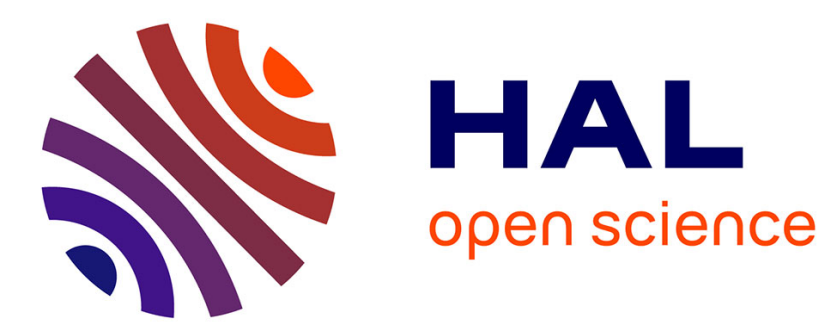

\title{
Frozen Boolean partial co-clones
}

Gustav Nordh, Bruno Zanuttini

\section{To cite this version:}

Gustav Nordh, Bruno Zanuttini. Frozen Boolean partial co-clones. Proc. 39th International Symposium on Multiple-Valued Logic (ISMVL 2009), May 2009, Japan. pp.120-125. hal-00944410

\section{HAL Id: hal-00944410 https://hal.science/hal-00944410}

Submitted on 12 Feb 2014

HAL is a multi-disciplinary open access archive for the deposit and dissemination of scientific research documents, whether they are published or not. The documents may come from teaching and research institutions in France or abroad, or from public or private research centers.
L'archive ouverte pluridisciplinaire HAL, est destinée au dépôt et à la diffusion de documents scientifiques de niveau recherche, publiés ou non, émanant des établissements d'enseignement et de recherche français ou étrangers, des laboratoires publics ou privés. 


\title{
Frozen Boolean partial co-clones
}

\author{
Gustav Nordh* $\quad$ Bruno Zanuttini ${ }^{\dagger}$
}

\begin{abstract}
We introduce and investigate the concept of frozen partial co-clones. Our main motivation for studying frozen partial co-clones is that they have important applications in complexity analysis of constraints. The frozen partial coclones lie between the co-clones and partial co-clones in the sense that the partial co-clone lattice is a refinement of the frozen partial co-clone lattice, which in turn is a refinement of the co-clone lattice. We concentrate on the Boolean domain and determine large parts of the frozen partial coclone lattice.
\end{abstract}

\section{Introduction}

A clone is a composition closed set of operations containing all projections. An operation $f$ preserves a relation $R$ (or is a polymorphism of $R$ ) if $f$ applied coordinate-wise to any tuples from $R$ gives a tuple in $R$. Given a set of relations $\Gamma, \operatorname{Pol}(\Gamma)$ is the set of operations preserving all relations in $\Gamma . \operatorname{Pol}(\Gamma)$ is always a clone and any clone can be defined as $\operatorname{Pol}(\Gamma)$ for a set of relations $\Gamma$. $\operatorname{Inv}(F)$ is the set of relations that are preserved by (invariant under) all the operations in $F$.

An $n$-ary relation $R$ has a primitive positive (p.p.) definition (also called an implementation) in a set of relations $\Gamma$ if $R$ is the set of models of an existentially quantified conjunction of atomic formulas over $\Gamma \cup\{=\}$ (also called a $\Gamma$-formula), i.e., $R\left(x_{1}, \ldots, x_{n}\right) \equiv \exists X \bigwedge_{i} R_{i}\left(x_{i 1}, \ldots, x_{i t}\right)$ where each $R_{i} \in \Gamma \cup\{=\}$. Sets of relations closed under p.p. definability are called co-clones and the least co-clone containing $\Gamma$ is denoted by $\langle\Gamma\rangle$. There is a Galois connection between clones and sets of relations closed under p.p. definability (i.e., co-clones). In particular, given two sets of relations $\Gamma_{1}$ and $\Gamma_{2}$, then $\left\langle\Gamma_{1}\right\rangle \subseteq\left\langle\Gamma_{2}\right\rangle$ if and only if $\operatorname{Pol}\left(\Gamma_{2}\right) \subseteq \operatorname{Pol}\left(\Gamma_{1}\right)$, and for any set of relations $\Gamma$ we have $\langle\Gamma\rangle=\operatorname{Inv}(\operatorname{Pol}(\Gamma))$. For more information on clones, co-

${ }^{*}$ IDA, Linköpings Universitet, gusno@ida. liu.se. Partially supported by the Swedish-French foundation.

${ }^{\dagger}$ GREYC, UMR CNRS 6072, Université de Caen Basse-Normandie, ENSICAEN, bruno.zanuttini@info.unicaen.fr. Supported by ANR grant CANAR (ANR-06-BLAN-0383-02). clones, and the aforementioned Galois connection, we refer the reader to the books $[14,16]$.

The $\operatorname{CSP}(\Gamma)$ problem, where $\Gamma$ is a set of relations (also called a constraint language), is the problem of deciding if a given set of variables subject to a set of constraints (given by atomic formulas over $\Gamma$ ) is satisfiable. The problem of classifying the computational complexity of $\operatorname{CSP}(\Gamma)$ with respect to $\Gamma$ is an important open problem. The (so far) most successful approach to attack this problem is an algebraic approach which heavily relies on the following result.

Theorem $1([11,12])$ If $\Gamma_{1}$ is finite and $\left\langle\Gamma_{1}\right\rangle \subseteq\left\langle\Gamma_{2}\right\rangle$, then $\operatorname{CSP}\left(\Gamma_{1}\right)$ is polynomial-time reducible to $\operatorname{CSP}\left(\Gamma_{2}\right)$.

Note that because of the Galois connection between clones and co-clones this result can be reformulated into: If $\Gamma_{1}$ is finite and $\operatorname{Pol}\left(\Gamma_{2}\right) \subseteq \operatorname{Pol}\left(\Gamma_{1}\right)$, then $\operatorname{CSP}\left(\Gamma_{1}\right)$ is polynomial-time reducible to $\operatorname{CSP}\left(\Gamma_{2}\right)$. To illustrate the power of this result, note that Schaefer's [18] seminal complexity classification (separating the cases in $\mathrm{P}$ from the NP-complete cases) for $\operatorname{CSP}(\Gamma)$ over the Boolean domain follows trivially from this result and Post's classification of Boolean clones [15] (i.e., Post's lattice). For more information on the connection between clones, co-clones, and the $\operatorname{CSP}(\Gamma)$ problem, we refer the reader to the survey articles $[3,4,13]$.

This result is not so useful for more fine grained complexity analysis of $\operatorname{CSP}(\Gamma)$ (and similar) problems. The reason is that it does not preserve the size of the problem instances (in terms of the number of variables). The crux is that in order for the proof of Theorem 1 to work, the existential quantifiers in p.p. definitions are eliminated by introducing new variables. So the reduction in Theorem 1 maps a conjunction of $m$ constraints on $n$ variables to a conjunction of $b m$ constraints on $n+a m$ variables, where $a$ and $b$ are constants which depend on the languages $\Gamma_{1}$ and $\Gamma_{2}$.

As an example of a problem for which this is too coarse a reduction, compare 3-SAT (i.e., $\operatorname{CSP}\left(\Gamma_{3 S A T}\right)$ where $\Gamma_{3 S A T}$ consists of the relations corresponding to clauses on at most three variables) with 1-in-3-SAT (i.e., $\operatorname{CSP}\left(\Gamma_{1 / 3}\right)$ where $\Gamma_{1 / 3}$ consists of the relation $\left.\{001,010,100\}\right)$. By Post's lattice it is easy to verify that $\left\langle\Gamma_{1 / 3}\right\rangle=\left\langle\Gamma_{3 S A T}\right\rangle$ and hence, $\operatorname{CSP}\left(\Gamma_{3 S A T}\right)$ is polynomial-time equivalent to 
$\operatorname{CSP}\left(\Gamma_{1 / 3}\right)$ according to Theorem 1. Despite this, 3-SAT (solvable in time $O\left(1.473^{n}\right)[1,7]$ ) seems to be a much harder problem than 1-in-3-SAT which can be solved in time $O\left(1.1003^{n}\right)$ [2] (where $n$ is the number of variables). Hence, it is clear that to get a better understanding of the complexity of $\operatorname{CSP}(\Gamma)$ (and similar) problems, we need reductions/implementations/tools where the blow-up in instance size can be controlled.

With this in mind we consider partial co-clones instead. A partial clone is a composition closed set of partial functions containing all (total) projections. A partial operation $f$ preserves a relation $R$ (or is a partial polymorphism of $R$ ) if $f$ applied coordinate-wise to any tuples from $R$ gives a tuple in $R$ whenever $f$ is defined on all the arguments. $p \operatorname{Pol}(\Gamma)$ is the set of (partial) operations preserving all relations in $\Gamma$. Partial co-clones can be defined as the sets of relations that are closed under p.p. definitions not using existential quantification, and the least partial co-clone containing $\Gamma$ is denoted by $\langle\Gamma\rangle_{p}$. There is also a Galois connection between partial co-clones and partial polymorphisms, in particular we have the following result.

Theorem $2([9,8,17])$ Let $\Gamma_{1}$ and $\Gamma_{2}$ be sets of relations. Then $\left\langle\Gamma_{1}\right\rangle_{p} \subseteq\left\langle\Gamma_{2}\right\rangle_{p}$ if and only if pPol $\left(\Gamma_{2}\right) \subseteq p \operatorname{Pol}\left(\Gamma_{1}\right)$.

For formal definitions and more information on partial clones and partial co-clones we refer the reader to [14].

Since partial co-clones do not utilize existential quantification, it can be verified that if we replace co-clones by partial co-clones in Theorem 1 then the reductions are sizepreserving (in terms of the number of variables). Hence, if $\left\langle\Gamma_{1}\right\rangle_{p} \subseteq\left\langle\Gamma_{2}\right\rangle_{p}$ and $\Gamma_{1}$ is finite then $\operatorname{CSP}\left(\Gamma_{1}\right)$ is solvable in time $O(f(n))$ if $\operatorname{CSP}\left(\Gamma_{2}\right)$ is solvable in time $O(f(n))$ (where $n$ is the number of variables). This result seems more useful than what it is since the structure of partial coclones is very complicated and only small portions of the lattice are known even for the Boolean domain [10]. We remark in passing that there are other applications of partial clones in complexity analysis of CSP( $\Gamma)$ problems [19].

What we would like to have is a concept that combines the good features of partial co-clones (e.g., size-preserving reductions for $\operatorname{CSP}(\Gamma))$ and the good features of co-clones (e.g., simpler structure especially over the Boolean domain). In this paper we propose and investigate such a concept that we call frozen partial co-clones. Frozen partial co-clones can be defined as the sets of relations that are closed under p.p. definitions where only the variables that take the same value in every model of the p.p. definition/formula (so called frozen variables) can be existentially quantified. The least frozen partial co-clone containing $\Gamma$ is denoted $\langle\Gamma\rangle_{f r}$. Hence, the frozen partial co-clones lie between the co-clones and partial co-clones in the sense that the partial co-clone lattice is a refinement of the frozen partial co-clone lattice, which in turn is a refinement of the co- clone lattice. Moreover, if we replace co-clones by frozen partial co-clones in Theorem 1 then it can be verified that if $\left\langle\Gamma_{1}\right\rangle_{f r} \subseteq\left\langle\Gamma_{2}\right\rangle_{f r}$ and $\Gamma_{1}$ is finite then $\operatorname{CSP}\left(\Gamma_{1}\right)$ is solvable in time $O(f(n+|D|))$ if $\operatorname{CSP}\left(\Gamma_{2}\right)$ is solvable in time $O(f(n))$ (where $D$ is the domain). The point is that all the variables that are frozen to the same domain element can be replaced by a single variable, and hence, at most $|D|$ extra variables need to be introduced when eliminating the existential quantifiers.

In this paper we focus exclusively on the Boolean domain. As expected, there is a connection between frozen partial co-clones and partial polymorphisms that we sketch (for the Boolean domain) in Section 2. In Sections 3 and 4 we determine large portions of the frozen (Boolean) partial co-clone lattice which indeed is significantly simpler than the partial co-clone lattice.

\section{Frozen partial co-clones and partial poly- morphisms}

In this section we give some definitions and preliminary remarks before sketching the connection between frozen (Boolean) partial co-clones and frozen (Boolean) partial polymorphisms. If $\varphi$ is a formula, then $\operatorname{Vars}(\varphi)$ denotes the set of variables occurring in it, and $\mathcal{M}(\varphi)$ denotes the set of all assignments to $\operatorname{Var} s(\varphi)$ which satisfy $\varphi$ (i.e., the models of $\varphi$ ). The relations $\{0\}$ and $\{1\}$ are denoted by $F$ and $T$, respectively. Atomic formulas are sometimes written in prefix notation (e.g., $R\left(x_{1}, \ldots, x_{n}\right)$ ), or infix notation (e.g., $x_{1} \neq x_{2}$ ), depending on the context. Given a function $f, \operatorname{dom}(f)$ denotes the domain of $f$ (i.e., the set of tuples $t_{i}$ for which $f\left(t_{i}\right)$ is defined), and $f$ is a subfunction of $g$ if $\operatorname{dom}(f) \subseteq \operatorname{dom}(g)$ and $f\left(t_{i}\right)=g\left(t_{i}\right)$ for all $t_{i} \in \operatorname{dom}(f)$.

Definition 3 (frozen variable) Let $\varphi$ be a formula and let $x \in \operatorname{Vars}(\varphi)$. Then $x$ is said to be frozen in $\varphi$ if $\varphi \models$ $T(x)$ or $\varphi \models F(x)$. In other words, $x$ is frozen in $\varphi$ if it is assigned the same value by all its models.

Definition 4 (frozen implementation) Let $\Gamma$ be a set of relations and $R$ an $n$-ary relation. Then $\Gamma$ freezingly implement $R$ if there is a p.p. definition $R\left(x_{1}, \ldots, x_{n}\right) \equiv$ $\exists X \varphi$ such that $\varphi$ is a conjunction of atomic formulas over $\Gamma \cup\{=\}, \operatorname{Var} s(\varphi) \subseteq X \cup\left\{x_{1}, \ldots, x_{n}\right\}$, and every variable in $X$ is frozen in $\varphi$.

Note that frozen implementations are slightly less general than so-called faithful implementations [5, page 34]. But the latter are not suitable for our purposes since they blow up instance sizes.

Definition 5 (frozen partial co-clone) Let $\Gamma$ be a set of relations. The frozen partial co-clone generated by $\Gamma$, written 
$\langle\Gamma\rangle_{f r}$, is the set of all relations that can be freezingly implemented by $\Gamma$. $\Gamma$ is also called a frozen basis of $\langle\Gamma\rangle_{f r}$.

It is easy to see that frozen implementations compose together, in the sense that if $\Gamma$ freezingly implements every relation in $\Gamma^{\prime}$ and $\Gamma^{\prime}$ freezingly implements $R$, then $\Gamma$ freezingly implements $R$. It is also easy to see that frozen partial co-clones ordered by set inclusion form a lattice.

Example 6 The relation $R^{\prime}=\{00,10\}$ is in $\langle\{R, T\}\rangle_{f r}$ with $R=\{000,001,110\}$ and $T=\{1\}$. This is because $R^{\prime}\left(x_{1}, x_{2}\right) \equiv \exists x_{3} R\left(x_{2}, x_{2}, x_{3}\right) \wedge R\left(x_{2}, x_{2}, x_{1}\right) \wedge T\left(x_{3}\right)$ where $x_{3}$ is frozen to 1 .

As another example, consider the relations $R_{1}=$ $\{01,10\}$ and $R_{2}=\{0100,0110,1000,1111\}$. Then $R^{\prime}=\{010,011,100\}$ is in $\left\langle\left\{R_{1}, R_{2}\right\}\right\rangle_{f r}$, as shown by $R^{\prime}\left(x_{1}, x_{2}, x_{3}\right) \equiv \exists x_{4} R_{1}\left(x_{1}, x_{2}\right) \wedge R_{2}\left(x_{1}, x_{2}, x_{3}, x_{4}\right)\left(x_{4}\right.$ is frozen to 0 by the conjunction).

Definition 7 (determined) If $\Gamma$ is a set of relations such that there is a $\Gamma$-formula $\varphi$ in which $x$ is frozen to $d \in$ $\{0,1\}$, then we say that $d$ is determined in $\Gamma$.

Proposition 8 Let $\Gamma$ be a set of relations. Then $d \in\{0,1\}$ is determined in $\Gamma$ if and only if $\{d\} \in\langle\Gamma\rangle_{f r}$.

Proof: Without loss of generality assume $d=1$. If 1 is determined in $\Gamma$, then there is a $\Gamma$-formula $\varphi$ and a variable $x_{T} \in \operatorname{Vars}(\varphi)$ such that $\exists X \varphi \models T\left(x_{T}\right)$ and thus $\varphi \models$ $T\left(x_{T}\right)$. Let $m$ be a model of $\varphi$ with a maximum number of variables being assigned 1 . Identify all variables in $\varphi$ that are assigned 1 by $m$ to $x_{T}$, resulting in $\varphi^{\prime}$. If $\operatorname{Vars}\left(\varphi^{\prime}\right)=$ $\left\{x_{T}\right\}$, then $T\left(x_{T}\right) \equiv \varphi^{\prime}$, and thus $T \in\langle\Gamma\rangle_{f r}$. Otherwise, there is a variable $x_{F} \in \operatorname{Vars}\left(\varphi^{\prime}\right) \backslash\left\{x_{T}\right\}$. Identify all variables in $\operatorname{Vars}\left(\varphi^{\prime}\right) \backslash\left\{x_{T}\right\}$ to $x_{F}$, resulting in $\varphi^{\prime \prime}$. Then, $T\left(x_{T}\right) \equiv \exists x_{F} \varphi^{\prime \prime}$, and $T \in\langle\Gamma\rangle_{f r}$ since $x_{F}$ is frozen in $\varphi^{\prime \prime}$.

The converse follows directly from the definition of determined constants.

Definition 9 (frozen partial polymorphisms) A $k$-ary (partial) function $f \in p \operatorname{Pol}(\Gamma)$ is said to be frozen if it is defined on every all- $d$-tuple (of length $k$ ) for which $d$ is determined in $\Gamma$ and $f(d, d, \ldots, d)=d$. We define $\operatorname{frPol}(\Gamma)=\{f \in \operatorname{pPol}(\Gamma) \mid f$ is frozen $\}$, and say that $\operatorname{frPol}(\Gamma)$ are the frozen polymorphisms of $\Gamma$.

Note that if no $d$ is determined in $\Gamma$, then $p \operatorname{Pol}(\Gamma)=$ $\operatorname{frPol}(\Gamma)$ and $\langle\Gamma\rangle_{p}=\langle\Gamma\rangle_{f r}$.

Lemma 10 Let $\Gamma$ be a frozen partial co-clone, then any $k$ ary (partial) function $f \in p \operatorname{Pol}(\Gamma)$ is a subfunction of a $k$-ary (partial) function $g \in \operatorname{frPol}(\Gamma)$.
Proof: It is sufficient to prove that if $\Gamma$ is a frozen partial co-clone such that $d \in\{0,1\}$ is determined in $\Gamma$, then any $f \in p \operatorname{Pol}(\Gamma)$ is a subfunction of a $g \in p \operatorname{Pol}(\Gamma)$ such that $\mathbf{d}=(d, d, \ldots, d) \in \operatorname{dom}(g)$ and $g(d, d, \ldots, d)=d$. Note that there can be no $f \in p \operatorname{Pol}(\Gamma)$ such that $f(d, \ldots, d) \neq d$ since this contradicts the fact (observed in Proposition 8) that $\{d\} \in \Gamma$.

Let $f \in p \operatorname{Pol}(\Gamma)$ be a $k$-ary function with $\operatorname{dom}(f)=$ $\left\{t_{1}, \ldots, t_{j}\right\}$ such that the $k$-tuple $\mathbf{d}=(d, \ldots, d)$ is not in $\operatorname{dom}(f)$. We define the function $f_{d}$ with $\operatorname{dom}\left(f_{d}\right)=$ $\operatorname{dom}(f) \cup\{\mathbf{d}\}$ such that $f_{d}(\mathbf{d})=d$ with the goal of showing that $f_{d} \in \operatorname{pPol}(\Gamma)$. Assume to the contrary that there is a relation $R_{d} \in \Gamma$ such that $R_{d}$ is not preserved by $f_{d}$. This means that there are $k$ (not necessarily distinct) tuples $t_{1}, \ldots, t_{k} \in R_{d}$ such that $\left(f_{d}\left(t_{1}[1], \ldots, t_{k}[1]\right), \ldots, f_{d}\left(t_{1}[m], \ldots, t_{k}[m]\right)\right) \notin R_{d}$ where $m$ is the arity of $R_{d}$. By the definition of $f_{d}$ and the fact that $f \in p \operatorname{Pol}(\Gamma)$ we know that at least one of the tuples $\left(t_{1}[j], \ldots, t_{k}[j]\right)=\mathbf{d}$. We can without loss of generality assume an $R_{d}$ such that $\left(t_{1}[1], \ldots, t_{k}[1]\right)=\mathbf{d}$ and all other $\left(t_{1}[j], \ldots, t_{k}[j]\right) \neq \mathbf{d}$.

Since $\{d\} \in \Gamma$ we have that $R\left(y_{1}, \ldots, y_{m-1}\right) \equiv$ $\exists x R_{d}\left(x, y_{1}, \ldots, y_{m-1}\right) \wedge(x=d)$ is in $\Gamma$ and thus is preserved by $f$. As a consequence, we have $\left(f\left(t_{1}[2], \ldots, t_{k}[2]\right), \ldots, f\left(t_{1}[m], \ldots, t_{k}[m]\right)\right) \in R$ and hence, $\left(d, f\left(t_{1}[2], \ldots, t_{k}[2]\right), \ldots, f\left(t_{1}[m], \ldots, t_{k}[m]\right)\right)=$ $\left(f_{d}\left(t_{1}[1], \ldots, t_{k}[1]\right), \ldots, f_{d}\left(t_{1}[m], \ldots, t_{k}[m]\right)\right) \in R_{d}$ and we have a contradiction. Thus, $f_{d} \in p \operatorname{Pol}(\Gamma)$.

Proposition 11 Given a set of relations $\Gamma$, then $\operatorname{fr} \operatorname{Pol}(\Gamma)=f r \operatorname{Pol}\left(\langle\Gamma\rangle_{f r}\right)$

Proof: $\quad$ Since $\Gamma \subseteq\langle\Gamma\rangle_{f r}$ we obviously have $\operatorname{fr} \operatorname{Pol}\left(\langle\Gamma\rangle_{f r}\right) \subseteq \operatorname{frPol}(\Gamma)$. For the other direction, assume towards contradiction that $f \in \operatorname{frPol}(\Gamma)$ but $f \notin$ $\operatorname{fr} \operatorname{Pol}\left(\langle\Gamma\rangle_{f r}\right)$. Then there is a relation $R \in\langle\Gamma\rangle_{f r}$ such that $f \notin \operatorname{frPol}(R)$ and a $\Gamma$-formula $\varphi$ such that $R \equiv \exists X \varphi$ where the variables $X$ are frozen in $\varphi$. This is a contradiction since $f \in \operatorname{frPol}(\Gamma)$ implies that $f \in \operatorname{frPol}(\mathcal{M}(\varphi))$ and hence $f \in \operatorname{frPol}(R)$ (since $f$ is frozen).

Theorem 12 Let $\Gamma_{1}$ and $\Gamma_{2}$ be sets of relations, then $\left\langle\Gamma_{1}\right\rangle_{f r} \subseteq\left\langle\Gamma_{2}\right\rangle_{f r}$ if and only if $\operatorname{frPol}\left(\Gamma_{2}\right) \subseteq \operatorname{frPol}\left(\Gamma_{1}\right)$.

Proof: If there is a (partial) function $f$ such that $f \in$ $\operatorname{frPol}\left(\Gamma_{2}\right)$ and $f \notin \operatorname{frPol}\left(\Gamma_{1}\right)$, then assume (with the aim of reaching a contradiction) that $\left\langle\Gamma_{1}\right\rangle_{f r} \subseteq\left\langle\Gamma_{2}\right\rangle_{f r}$. From the fact that $f \in \operatorname{frPol}\left(\Gamma_{2}\right)$ and $f \notin \operatorname{frPol}\left(\Gamma_{1}\right)$ we get that there is a relation $R \in \Gamma_{1}$ such that $R \notin \Gamma_{2}$ and $f \notin p \operatorname{Pol}(R)$. By assumption $\left\langle\Gamma_{1}\right\rangle_{f r} \subseteq\left\langle\Gamma_{2}\right\rangle_{f r}$, and hence there is a frozen implementation of $R$ using the relations in $\Gamma_{2}$. So, there is a $\Gamma_{2}$-formula $\varphi$ such that $R \equiv \exists X \varphi$ where the variables in $X$ are frozen in $\varphi$. But $\varphi$ is a $\Gamma_{2}$-formula 


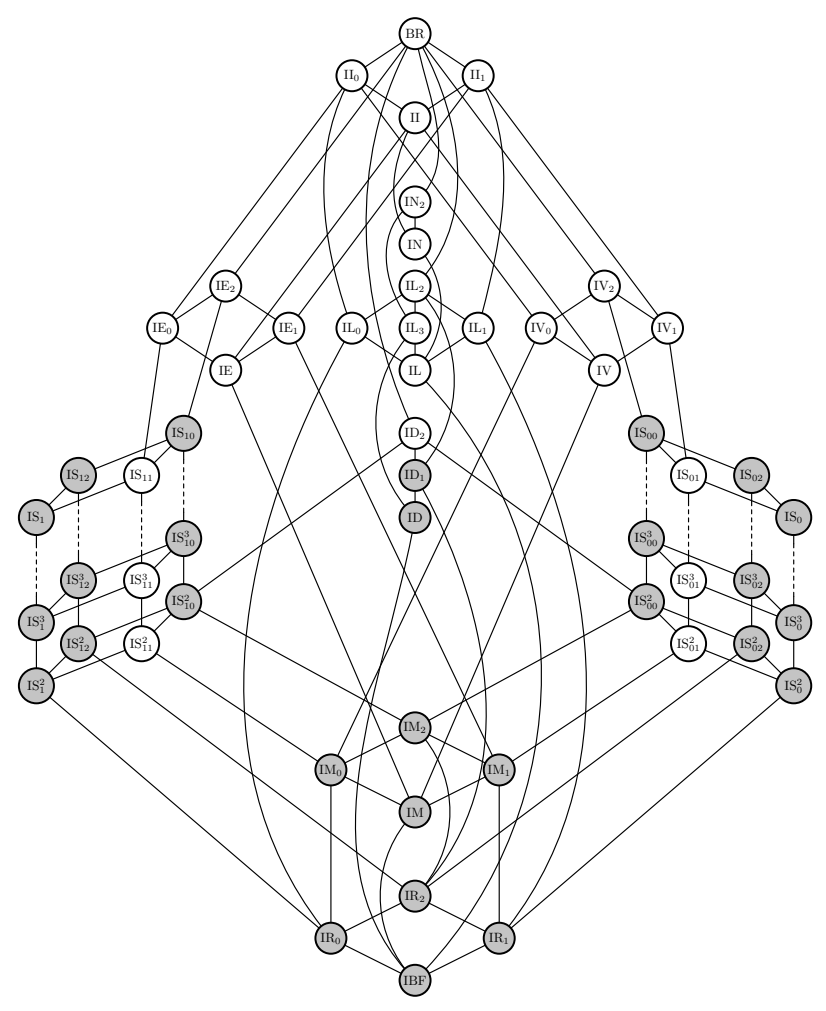

Figure 1. The lattice of Boolean co-clones.

and $f \in \operatorname{frPol}\left(\Gamma_{2}\right)$, so $f \in \operatorname{pPol}(\mathcal{M}(\varphi))$. Moreover, as a frozen polymorphism of $\Gamma_{2}, f$ is defined on all columns of $\mathcal{M}(\varphi)$ corresponding to variables $X$ (Proposition 8), and finally $f \in p \operatorname{Pol}(R)$.

Now we continue with the other direction. If $\left\langle\Gamma_{1}\right\rangle_{f r} \not \mathbb{E}$ $\left\langle\Gamma_{2}\right\rangle_{f r}$, then since obviously $\left\langle\langle\Gamma\rangle_{f r}\right\rangle_{p}=\langle\Gamma\rangle_{f r}$ for any language $\Gamma$, we have $\left.\left\langle\left\langle\Gamma_{1}\right\rangle_{f r}\right\rangle_{p} \nsubseteq \not\left\langle\Gamma_{2}\right\rangle_{f r}\right\rangle_{p}$. Hence, by Theorem 2 there exists a (partial) function $f^{\prime}$ such that $f^{\prime} \in p \operatorname{Pol}\left(\left\langle\Gamma_{2}\right\rangle_{f r}\right)$ and $f^{\prime} \notin p \operatorname{Pol}\left(\left\langle\Gamma_{1}\right\rangle_{f r}\right)$. By Lemma 10, we have that $f^{\prime}$ is a subfunction of a (partial) function $f \in \operatorname{frPol}\left(\left\langle\Gamma_{2}\right\rangle_{f r}\right)$. It is clear that $f \notin f r \operatorname{Pol}\left(\left\langle\Gamma_{1}\right\rangle_{f r}\right)$ since not even the subfunction $f^{\prime}$ is in $\operatorname{pPol}\left(\left\langle\Gamma_{1}\right\rangle_{f r}\right)$. Hence, by Proposition 11, we get $f \in \operatorname{frPol}\left(\Gamma_{2}\right)$ and $f \notin \operatorname{frPol}\left(\Gamma_{1}\right)$.

Corollary 13 Let $\Gamma$ be a set of relations. Then $\operatorname{Inv}(\operatorname{frPol}(\Gamma))=\langle\Gamma\rangle_{f r}$.

\section{Co-clones covered by a single frozen partial co-clone}

In this section we study Boolean co-clones $C$ such that for any set of relations $\Gamma$ with $\langle\Gamma\rangle=C$ we have $C=\langle\Gamma\rangle_{f r}$. We say that such a co-clone is covered by a single frozen partial co-clone. We show that a large number of co-clones are covered by a single frozen partial co-clone, and hence a large part of the lattice of frozen partial co-clones is identical to the corresponding part of the lattice of co-clones. The lattice of Boolean co-clones is visualised in Figure 1, where the co-clones colored grey are covered by a single frozen partial co-clone. For explanations of the notation ${ }^{1}$ used in the lattice, we refer the reader to $[3,4]$.

We define the most relevant co-clones here. A majority operation is a ternary operation $m a j$ satisfying $\operatorname{maj}(x, x, y)=\operatorname{maj}(x, y, x)=\operatorname{maj}(y, x, x)=x$ for all $x, y \in\{0,1\}$. Similarly a minority operation is a ternary operation minor satisfying minor $(x, x, y)=$ $\operatorname{minor}(x, y, x)=\operatorname{minor}(y, x, x)=y$ for all $x, y \in$ $\{0,1\}$. The binary operations $\max$ and $\min$ return the maximum and minimum of their arguments, respectively. The co-clones $I D_{2}, I D_{1}$, and $I M_{2}$ are defined as follows: $I D_{2}=\operatorname{Inv}(\{m a j\}), I D_{1}=\operatorname{Inv}(\{m a j$, minor $\})$, and $I M_{2}=\operatorname{Inv}(\{\max , \min \})$.

In [10] it is proved that there are 25 partial co-clones ${ }^{2}$ $p C$ such that $p C \subseteq I M_{2}=\operatorname{Inv}(\{\max , \min \})$, and there are 33 partial co-clones $p C$ such that $p C \subseteq I D_{1}=$ $\operatorname{Inv}(\{$ maj, minor $\})$. Since all co-clones $C$ such that $C \subseteq$ $I M_{2}$ or $C \subseteq I D_{1}$ are covered by a single frozen partial coclone, there are only 8 frozen partial co-clones $f C \subseteq I M_{2}$ and 6 frozen partial co-clones $f C \subseteq I D_{1}$. This suggests that the lattice of frozen partial co-clones is significantly less complex than the partial co-clone lattice. Nevertheless, as expected the frozen partial co-clone lattice is more complex than the ordinary co-clone lattice. In particular we show in Section 4 that the co-clone $I D_{2}$ splits into 13 frozen partial co-clones. Moreover, it seems that none of the white co-clones in Figure 1 is covered by a single frozen partial co-clone.

The covering proofs make heavy use of the results in [6] which for every Boolean co-clone $C$ gives a set of relations $\Gamma$ such that $\langle\Gamma\rangle_{p}=C$. In particular, it is shown in [6] that $I D_{1}=\langle\{\neq, T, F\}\rangle_{p}$. The covering proofs are numerous so due to space constraints we can only present a single illustrative case.

Proposition $14 I D_{1}=\operatorname{Inv}(\{$ maj,minor $\})=$ $\langle\{\neq, T, F\}\rangle_{p}$ is covered by a single frozen partial coclone.

Proof: Let $\Gamma \subseteq I D_{1}$ with $\Gamma \nsubseteq I D$ and $\Gamma \nsubseteq I R_{2}$ (i.e., $\left.\langle\Gamma\rangle=I D_{1}\right)$, where $I D=\langle\neq\rangle$ and $I R_{2}=\langle\{F, T\}\rangle$. First note that since $\langle\Gamma\rangle=I D_{1}=\langle\{\neq, T, F\}\rangle$, we trivially have that $F$ and $T$ are determined in $\Gamma$. Thus, according to Proposition 8 we have $\{T, F\} \subseteq\langle\Gamma\rangle_{f r}$.

Now, since $\Gamma \nsubseteq I R_{2}$ there is a relation $R \in \Gamma$ such that

\footnotetext{
${ }^{1}$ The notation used is different from Post's notation, but is now standard in the Boolean CSP area.

${ }^{2}$ The results in [10] are presented in terms of partial clones.
} 
$R \notin I R_{2}$. Then there is $\varphi \equiv R$ of the form:

$$
\bigwedge_{i \in I}\left(x_{i} \neq y_{i}\right) \wedge \bigwedge_{j \in J} F\left(x_{j}\right) \wedge \bigwedge_{k \in K} T\left(x_{k}\right)
$$

Assume no $j$ and no $k$ is in $I$, which can be ensured by propagating unary constraints. From $R \notin I R_{2}$ we know that $R$ is nonempty and $I \neq \emptyset$. Let $m \in R$, that is, a model of $\varphi$, and let $P=\left\{x_{i} \mid i \in I, m\left(x_{i}\right)=1\right\} \cup\left\{y_{i} \mid i \in\right.$ $\left.I, m\left(y_{i}\right)=1\right\}$ and $N=\left\{x_{i} \mid i \in I, m\left(x_{i}\right)=0\right\} \cup\left\{y_{i} \mid\right.$ $\left.i \in I, m\left(y_{i}\right)=0\right\}$. Because $I \neq \emptyset$ we have at least one $\{\neq\}$-constraint and so, $P, N \neq \emptyset$. Moreover, obviously every $\{\neq\}$-constraint in $\varphi$ is between a variable in $P$ and one in $N$. Now identify all the variables in $P$ to a single variable $p$ and all those in $N$ to $n$. Then clearly the resulting formula is logically equivalent to $(p \neq n) \wedge \bigwedge_{j \in J} F\left(x_{j}\right) \wedge$ $\bigwedge_{k \in K} T\left(x_{k}\right)$, and we get a frozen implementation of $\neq$ by existentially quantifying over every $x_{j}$ and $x_{k}(j \in J, k \in$ $K)$.

Finally, $\{\neq, T, F\} \subseteq\langle\Gamma\rangle_{f r}$ and so, $\langle\Gamma\rangle_{f r}=I D_{1}$.

Theorem 15 Each co-clone colored grey in Figure 1 is covered by a single frozen partial co-clone.

\section{Structure of $\mathrm{ID}_{2}$}

We begin by introducing the basic relations and the 13 frozen partial co-clones in $I D_{2}=\operatorname{Inv}(\{m a j\})$ (i.e., the frozen partial co-clones $\langle\Gamma\rangle_{f r}$ such that $\langle\Gamma\rangle_{f r} \subseteq I D_{2}$ and $\left.\langle\Gamma\rangle=I D_{2}\right)$. We then prove that these 13 frozen partial coclones cover $I D_{2}$ (i.e., $\langle\Gamma\rangle_{f r}$ equals one of these 13 frozen partial co-clones for any $\Gamma$ such that $\langle\Gamma\rangle=I D_{2}$ ). Finally, we prove that these 13 frozen partial co-clones are all distinct. We remark that the lattice of partial co-clones in $I D_{2}$ has not yet been classified [10]. Hence, the results in this section can also be seen as a step towards such a classification.

Definition 16 (relations in $\left.I D_{2}\right) R_{2}^{p}$ is the relation defined by $\left(x_{1} \vee x_{2}\right)$ (2 stands for binary and $p$ for positive). Similarly, $R_{2}^{n}$ is the relation defined by $\left(\neg x_{1} \vee \neg x_{2}\right), R_{2}^{i}$ is the relation defined by $\left(\neg x_{1} \vee x_{2}\right)$ ( $i$ stands for implicative), and $R_{2}^{\neq}$is the relation defined by $x_{1} \neq x_{2} . R_{3}^{p}$ is the relation defined by $\left(x_{1} \vee x_{2}\right) \wedge\left(x_{1} \neq x_{3}\right)$ and $R_{3}^{n}$ that defined by $\left(\neg x_{1} \vee \neg x_{2}\right) \wedge\left(x_{1} \neq x_{3}\right)$. Finally, $R_{4}^{p}$ is the relation defined by $\left(x_{1} \vee x_{2}\right) \wedge\left(x_{1} \neq x_{3}\right) \wedge\left(x_{2} \neq x_{4}\right)$.

Definition 17 [frozen partial co-clones in $I D_{2}$ ] We define the following frozen partial co-clones:

$$
\begin{aligned}
& \text { - } \Gamma_{4}^{p}=\left\langle R_{4}^{p}\right\rangle_{f r}, \\
& \text { - } \Gamma_{3}^{p}=\left\langle R_{3}^{p}\right\rangle_{f r}, \Gamma_{3}^{n}=\left\langle R_{3}^{n}\right\rangle_{f r},
\end{aligned}
$$

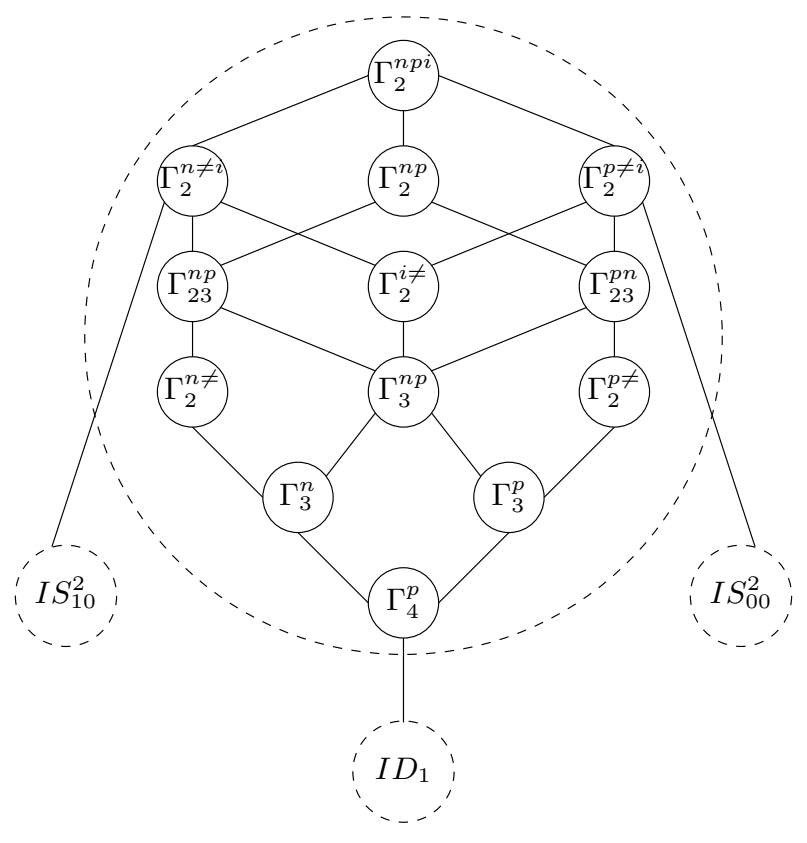

Figure 2. Frozen partial co-clones in $\mathrm{ID}_{2}$.

- $\Gamma_{3}^{n p}=\left\langle R_{3}^{n}, R_{3}^{p}\right\rangle_{f r}$,

- $\Gamma_{2}^{n \neq}=\left\langle R_{2}^{n}, R_{2}^{\neq}\right\rangle_{f r}, \Gamma_{2}^{p \neq}=\left\langle R_{2}^{p}, R_{2}^{\neq}\right\rangle_{f r}$,

- $\Gamma_{2}^{i \neq}=\left\langle R_{2}^{i}, R_{2}^{\neq}\right\rangle_{f r}, \Gamma_{2}^{n p}=\left\langle R_{2}^{n}, R_{2}^{p}\right\rangle_{f r}$,

- $\Gamma_{23}^{n p}=\left\langle R_{2}^{n}, R_{3}^{p}\right\rangle_{f r}, \Gamma_{23}^{p n}=\left\langle R_{2}^{p}, R_{3}^{n}\right\rangle_{f r}$,

- $\Gamma_{2}^{n \neq i}=\left\langle R_{2}^{n}, R_{2}^{\neq}, R_{2}^{i}\right\rangle_{f r}, \Gamma_{2}^{p \neq i}=\left\langle R_{2}^{p}, R_{2}^{\neq}, R_{2}^{i}\right\rangle_{f r}$,

- $\Gamma_{2}^{n p i}=\left\langle R_{2}^{n}, R_{2}^{p}, R_{2}^{i}\right\rangle_{f r}{ }^{3}$

The inclusion structure among these frozen partial coclones is given in Figure 2. Most of the inclusions are obvious. The main difficulty is to show that they cover all of $I D_{2}$. Due to space constraints and the fact that all the covering proofs are quite similar, we only present one of them. Note that the proof is similar in spirit to the constructions in [19].

Proposition $18\left(\Gamma_{4}^{p}\right)$ Let $R$ be a relation in $\Gamma_{4}^{p} \backslash I D_{1}$. Then $R$ freezingly implements $R_{4}^{p}$.

Proof: Given a relation $R \equiv \mathcal{M}(\varphi)$, then $R_{\mid\left\{x_{i}, \ldots, x_{j}\right\}}$ denotes the projection of $R$ onto the coordinates corresponding to the variables $\left\{x_{i}, \ldots, x_{j}\right\}$ in $\varphi$.

From $R \in \Gamma_{4}^{p}$ it follows that $R$ has a p.p. definition of the form $\exists X, \bigwedge_{i \in I} R_{4}^{p}\left(x_{i 1}, \ldots, x_{i 4}\right)$ where the variables in

\footnotetext{
${ }^{3}$ The mnemonics are: subscripts represent the arities of the relations in the basis, and superscripts represent the nature of these relations, in the same order ( $p$ stands for positive, etc.).
} 
$X$ are frozen. Write $R_{i}$ for $R_{\mid\left\{x_{i 1}, \ldots, x_{i 4}\right\}}$ and $R_{4}^{p}\left(X_{i}\right)$ for $R_{4}^{p}\left(x_{i 1}, \ldots, x_{i 4}\right)$. We claim that there is an $i_{0} \in I$ such that $R_{i_{0}}=R_{4}^{p}\left(X_{i_{0}}\right)$ and all $x_{i_{0} j}$ 's are pairwise different.

Assume to the contrary that for all $i, R_{i} \neq R_{4}^{p}\left(X_{i}\right)$. By construction it follows $R_{i} \subset R_{4}^{p}\left(X_{i}\right)$ (since at least the $\left\{R_{4}^{p}\right\}$-constraint acts on $\left.x_{i 1}, \ldots, x_{i 4}\right)$. But a case study on the tuples in $R_{4}^{p}\left(X_{i}\right) \backslash R_{i}$ shows that this entails $R_{i} \in I D_{1}$. Since from the definition of $R$ it follows $R \equiv \exists X, \bigwedge_{i \in I} R_{i}$, we get $R \in I D_{1}$, a contradiction.

Now consider the relation obtained from $R$ by applying the following transformations maximally while preserving $R_{i_{0}}=R_{4}^{p}\left(X_{i_{0}}\right)=\{0110,1001,1100\}:$

1. identify $x^{\prime}$ to $x$ for some $x \in\left\{x_{i_{0} 1}, \ldots, x_{i_{0}}\right\}$, $x^{\prime} \notin\left\{x_{i_{0} 1}, \ldots, x_{i_{0} 4}\right\}$; e.g., if $R_{\mid\left\{x_{i_{0} 1}, \ldots, x_{i_{0}}, x^{\prime}\right\}}=$ $\{01100,01101,10011,11001\}$, identify $x^{\prime}$ to $x_{i_{0} 1}$;

2. freeze $x^{\prime} \notin\left\{x_{i_{0} 1}, \ldots, x_{i_{0} 4}\right\}$ to 0 or 1 (using $F$ or $T$ with Proposition 8) and existentially quantify over it, e.g., if $R_{\mid\left\{x_{i_{0} 1}, \ldots, x_{i_{0} 4}, x^{\prime}\right\}}=$ $\{01100,01101,10010,11000\}$, freeze $x^{\prime}$ to 0 and existentially quantify over it.

When none of these operations can be applied any more, it is easily verified that all remaining $x^{\prime} \notin\left\{x_{i_{0} 1}, \ldots, x_{i_{0}}\right\}$ satisfy $R_{\mid\left\{x_{\left.i_{0} 1, \ldots, x_{i}, x^{\prime}\right\}}\right.}=\{01101,10011,11000\}$ or $R_{\mid\left\{x_{i_{0} 1}, \ldots, x_{i_{0}}, x^{\prime}\right\}}=\{01100,10010,11001\}$. But this is a contradiction, since then $R_{\mid\left\{x_{i_{0} 1}, \ldots, x_{i_{0}}, x^{\prime}\right\}}$ is not closed under ternary majority, and so $R \notin I D_{2}$. Thus the transformations end with $R=R_{4}^{p}\left(X_{i_{0}}\right)$ (no other $x_{j}$ can be left).

To conclude, from $R$ we freezingly implemented $R_{4}^{p}$, and we are done.

Theorem 19 Given any set of relations $\Gamma$ such that $\langle\Gamma\rangle=$ $I D_{2}$, then $\langle\Gamma\rangle_{f r}$ equals one of the 13 frozen partial coclones in Definition 17.

It is easy to prove that the 13 frozen partial co-clones in Definition 17 are all distinct. Given two sets of relations $\Gamma_{1}$ and $\Gamma_{2}$ such that $\left\langle\Gamma_{1}\right\rangle=\left\langle\Gamma_{2}\right\rangle=I D_{2}$, then to prove that $\left\langle\Gamma_{1}\right\rangle_{f r} \neq\left\langle\Gamma_{2}\right\rangle_{f r}$ it is sufficient (according to Theorem 12) to show that $\operatorname{frPol}\left(\Gamma_{2}\right) \neq \operatorname{frPol}\left(\Gamma_{1}\right)$.

The operations that we use to separate the different frozen partial co-clones are all ternary minority operations that are undefined on certain tuples. We denote these minority operations by $m U\left(t_{1}, \ldots, t_{n}\right)$ where $t_{1}, \ldots, t_{n}$ are the tuples on which the minority operation is undefined. For example, $\Gamma_{4}^{p} \neq \Gamma_{3}^{p}$ since $m U(010,001) \in \operatorname{frPol}\left(R_{4}^{p}\right)=$ $\operatorname{frPol}\left(\Gamma_{4}^{p}\right)$ but $m U(010,001) \notin \operatorname{frPol}\left(R_{3}^{p}\right)$, and $\Gamma_{3}^{p} \neq \Gamma_{2}^{p \neq}$ since $m U(100,010,001) \in \operatorname{frPol}\left(R_{3}^{p}\right)=$ $\operatorname{frPol}\left(\Gamma_{3}^{p}\right)$ but $m U(100,010,001) \notin \operatorname{frPol}\left(R_{2}^{p}\right)$.

Proposition 20 The 13 frozen partial co-clones in Definition 17 are all distinct.

\section{Acknowledgements}

We thank Henning and Ilka Schnoor and Magnus Wahlström for interesting discussions on the topic of this paper, and Steffen Reith for providing the visualisation of the lattice of Boolean co-clones in Figure 1.

\section{References}

[1] T. Brueggemann and W. Kern. An improved deterministic local search algorithm for 3-SAT. Theor. Comput. Sci., 329(1-3):303-313, 2004.

[2] J. M. Byskov, B. A. Madsen, and B. Skjernaa. New algorithms for exact satisfiability. Theor. Comput. Sci., 332(13):515-541, 2005.

[3] E. Böhler, N. Creignou, S. Reith, and H. Vollmer. Playing with Boolean blocks, part I: Post's lattice with applications to complexity theory. ACM SIGACT-Newsletter, 34(4):3852,2003

[4] E. Böhler, N. Creignou, S. Reith, and H. Vollmer. Playing with Boolean blocks, part II: Constraint satisfaction problems. ACM SIGACT-Newsletter, 35(1):22-35, 2004.

[5] N. Creignou, S. Khanna, and M. Sudan. Complexity classifications of Boolean constraint satisfaction problems. SIAM Monographs on Discrete Math. and Applications, 2001.

[6] N. Creignou, P. G. Kolaitis, and B. Zanuttini. Structure identification of Boolean relations and plain bases for co-clones. J. of Comput. and Syst. Sci., 74(7):1103-1115, 2008.

[7] E. Dantsin, A. Goerdt, E. Hirsch, R. Kannan, J. Kleinberg, C. Papadimitriou, P. Raghavan, and U. Schöning. A deterministic $(2-2 /(\mathrm{k}+1))^{\mathrm{n}}$ algorithm for $\mathrm{k}-\mathrm{SAT}$ based on local search. Theor. Comput. Sci., 289(1):69-83, 2002.

[8] I. Fleischner and I. G. Rosenberg. The Galois connection between partial operations and relations. Pacific J. Math., 79:93-97, 1978

[9] D. Geiger. Closed systems of functions and predicates. Pac. J. of Math., 27(1):95-100, 1968.

[10] L. Haddad and G. Simons. On intervals of partial clones of Boolean partial functions. In ISMVL, pages 315-320, 2003.

[11] P. Jeavons. On the algebraic structure of combinatorial problems. Theor. Comput. Sci., 200(1-2):185-204, 1998.

[12] P. Jeavons, D. Cohen, and M. Gyssens. Closure properties of constraints. J. of the ACM, 44:527-548, 1997.

[13] A. Krokhin, A. Bulatov, and P. Jeavons. Functions of multiple-valued logic and the complexity of constraint satisfaction: A short survey. In ISMVL, pages 343-351, 2003.

[14] D. Lau. Function Algebras on Finite Sets. Springer, Berlin, 2006.

[15] E. Post. The two-valued iterative systems of mathematical logic. Annals of Mathematical Studies, 5:1-122, 1941.

[16] R. Pöschel and L. Kaluznin. Funktionen- und Relationenalgebren. DVW, Berlin, 1979.

[17] B. A. Romov. The algebras of partial functions and their invariants. Cybernetics and Systems Analysis, 17(2):157$167,1981$.

[18] T. Schaefer. The complexity of satisfiability problems. In STOC, pages 216-226, 1978.

[19] H. Schnoor and I. Schnoor. New algebraic tools for constraint satisfaction. In Complexity of Constraints, 2006. 